\title{
The Prognosis and Recurrence Pattern of Right- and Left-Sided Colon Cancer in Stage II, Stage III, and Liver Metastasis After Curative Resection
}

\author{
Yasuyuki Nakamura, Daisuke Hokuto, Fumikazu Koyama, Yasuko Matsuo, Takeo Nomi, \\ Takahiro Yoshikawa, Naoki Kamitani, Tomomi Sadamitsu, Takeshi Takei, Yayoi Matsumoto, Yosuke Iwasa, \\ Kohei Fukuoka, Shinsaku Obara, Takayuki Nakamoto, Hiroyuki Kuge, Masayuki Sho \\ Department of Surgery, Nara Medical University, Kashihara, Japan
}

Purpose: Primary tumor location of colon cancer has been reported to affect the prognosis after curative resection. However, some reports suggested the impact was varied by tumor stage. This study analyzed the prognostic impact of the sidedness of colon cancer in stages II, III, and liver metastasis after curative resection using propensity-matched analysis.

Methods: Right-sided colon cancer was defined as a tumor located from cecum to splenic flexure, while any more distal colon cancer was defined as left-sided colon cancer. Patients who underwent curative resection at Nara Medical University hospital between 2000 and 2016 were analyzed.

Results: There were 110 patients with stage II, 100 patients with stage III, and 106 patients with liver metastasis. After propensity matching, 28 pairs with stage II and 32 pairs with stage III were identified. In the patients with stage II, overall survival (OS) and recurrence-free survival (RFS) were not significantly different for right- and left-sided colon cancers. In the patients with stage III, OS and RFS were significantly worse in right-sided colon cancer. In those with liver metastasis, OS of right-sided colon cancer was significantly worse than left-sided disease, while RFS was similar. Regarding metachronous liver metastasis, the difference was observed only in the patients whose primary colon cancer was stage III. In each stage, significantly higher rate of peritoneal recurrence was found in those with right-sided colon cancer.

Conclusion: Sidedness of colon cancer had a significant and varied prognostic impact in patients with stage II, III, and liver metastasis after curative resection.

Keywords: Right-sided colon cancer; Left-sided colon cancer; Colorectal liver metastasis

\section{INTRODUCTION}

Colorectal cancer is one of the most common cancers worldwide and is the fourth common cause of cancer-related deaths [1]. Recent studies have investigated the impact of primary tumor loca-

Received: Jun 12, 2020 - Revised: Sep 7, 2020 - Accepted: Sep 14, 2020 Correspondence to: Daisuke Hokuto, M.D.

Department of Surgery, Nara Medical University, 840 Shijo-cho, Kashihara, 634-8522 Nara, Japan

Tel: +81-744-29-8863, Fax: +81-0744-24-6866

E-mail: hokuto@naramed-u.ac.jp

ORCID: https://orcid.org/0000-0003-1661-6224

(C) 2021 The Korean Society of Coloproctology

This is an open-access article distributed under the terms of the Creative Commons Attribution NonCommercial License (https://creativecommons.org/licenses/by-nc/4.0) which permits unrestricted non-

commercial use, distribution, and reproduction in any medium, provided the original work is properly cited. tion on colon cancer. It was reported that right-sided colon cancer (RSC) more commonly displayed mucinous histologic characteristics, microsatellite instability (MSI), and mutations of RAS, BRAF, and PIK3CA genes compared with left-sided colon cancer (LSC) $[2,3]$. A meta-analysis of $1,437,846$ patients in 2017 reported that LSC was associated with a significantly reduced risk of death compared with RSC that was independent of tumor stage [4]. However, in 2019, another meta-analysis of 581,542 patients with stages I-III colon cancer reported that the prognosis of LSC was significantly better than RSC for stage III disease, while for stage I-II disease the prognosis of LSC was significantly worse than RSC [5]. Others also reported that the impact of tumor location on outcome varied by tumor stage $[6,7]$, and this is still a controversial topic.

The impact of primary tumor location of colorectal liver metastasis (CRLM) has been also studied. Analysis of patients with un- 
resectable CRLM who participated in 6 randomized trials (CRYSTAL, FIRE-3, CALGB 80405, PRIME, PEAK, and 20050181) showed worse overall survival (OS) and progression-free survival for patients with RSC [8]. For those with resectable CRLM, some studies reported that RSC was associated with worse OS and recurrence-free survival (RFS) $[9,10]$, while other studies found that although OS was worse in RSC, RFS was similar in both RSC and LSC $[11,12]$.

The aim of our study was to analyze the difference in clinicopathological features, patterns of recurrence, and prognostic impact according to the sidedness of the primary colon cancer in patients with stage II, III, and IV colon cancer with CRLM who underwent curative resection in a single center.

This study was approved by the Ethics Committee of Nara Medical University (No.1620, No.1986). Written informed consent for use of the patients' clinical data was obtained before surgery.

\section{METHODS}

\section{Study population}

For analysis of stage II and III colon cancer, the data of consecutive patients who underwent curative resection at the Department of Surgery, Nara Medical University in Kashihara, Japan between January 2007 and December 2013 were retrieved from a prospective database. Preoperative characteristics, operative outcomes, pathological findings, and long-term outcomes were analyzed separately according to the cancer stage. The stage was classified using the 7th edition of the Union for International Cancer Control/TNM classification. T and $\mathrm{N}$ factors were evaluated histologically. For analysis of CRLM, the data for all consecutive patients who underwent curative liver resection at the same institution between January 2000 and December 2016 were retrieved. Patients with rectal cancer were excluded. We divided the patients into 2 groups, namely those with RSC, defined as a tumor located between the cecum and the splenic flexure of the colon; and those with LSC, defined as a tumor located from the splenic flexure of the colon to the sigmoid colon. Clinicopathological factors included age, sex, $\mathrm{T}$ factor, $\mathrm{N}$ factor, lymphatic invasion, venous invasion, histological differentiation, carcinoembryonic antigen (CEA), carbohydrate antigen 19-9 (CA19-9), and use of adjuvant chemotherapy. For CRLM, details of the timing of liver metastasis (synchronous/metachronous), number of CRLM, and maximum tumor size of CRLM were also collected. Regarding stage II and III colon cancer, some clinicopathological factors were significantly different between the RSC group and the LSC group. Therefore, one-to-one propensity score matching analysis was performed. Long-term outcomes were evaluated by RFS and OS. Multivariate analysis using Cox proportional hazard model was also performed to analyze the prognostic impact of tumor sidedness.

\section{Patient management}

Regarding stage II and III colon cancer, all colectomies were car- ried out with curative intent and lymph node cleaning, the level determined by the attending surgeon's decision. In this study period, laparoscopic approach was performed mainly for T1 to T3 tumors. Bulky tumors or tumors with obvious lymph node swelling were applied for open colectomies. At least D2 lymph node dissection was performed in the all cases. D3 lymph node resection in LSC included the inferior mesenteric artery preserved cases and resected cases. Neoadjuvant chemotherapy was not performed in this study. Adjuvant chemotherapy was used in the patients with stage III or high-risk stage II colon cancer, if the general condition of the patient was compatible. In this study period, 6 months of uracil-tegafur/leucovorin (LV), S-1, or capecitabine was performed as adjuvant chemotherapy. Patients with numerous lymph node metastasis or T4 tumors underwent 6 months of FOLFOX (folinic acid, fluorouracil, and oxaliplatin) or XELOX (capecitabine and oxaliplatin). Regarding CRLM, all liver resections were carried out with curative intent. Neoadjuvant chemotherapy was not administered for resectable CRLM, while postoperative adjuvant chemotherapy was administered routinely, except in cases involving patients that exhibited a poor performance status or severe chronic renal failure. The main adjuvant chemotherapy regimen was arterial infusion chemotherapy or 5-fluorouracil/LV before 2004, while after 2005, oxaliplatin-based regimens such as FOLFOX, XELOX, and SOX (s-1 and oxaliplatin) were mainly used. Patients were followed-up every 4 months for up to 3 years after surgery and every 6 months thereafter. During the follow-up examinations, the patients' serum CEA and CA19-9 levels were measured and contrast-enhanced abdominal and thoracic computed tomography was performed. The sites of recurrence were recorded and were classified into liver, lung, peritoneum, lymph node, and other organs.

\section{Statistical analysis}

Baseline characteristics of patients were expressed as medians for continuous data, and absolute values and percentages for categorical data. Fisher exact test or the chi-square test was used to identify differences in the categorical variables. A survival analysis was conducted using the Kaplan-Meier method, and the significance of differences between survival curves was determined using the log-rank test. One-to-one propensity matching analyses for stage II and stage III colon cancer was performed. The propensity scores were calculated for each patient using logistic regression analysis involving the following covariates; age, sex, $\mathrm{T}$ factor, lymphatic invasion, and venous invasion. Univariate and multivariate analyses of prognostic factors using Cox proportional hazard model were performed for stage II and stage III colon cancer. Multivariate analysis was performed with the factors that were identified as significant prognostic factors in univariate analysis. All statistical analyses were performed using IBM SPSS Statistics ver. 26.0 (IBM Corp., Armonk, NY, USA), and statistical significance was defined as a $\mathrm{P}$ value of $<0.05$. 
Table 1. Characteristics of the patients with stage II colon cancer

\begin{tabular}{|c|c|c|c|c|c|c|}
\hline \multirow{2}{*}{ Variable } & \multicolumn{3}{|c|}{ Before propensity matching } & \multicolumn{3}{|c|}{ After propensity matching } \\
\hline & Right-sided $(n=56)$ & Left-sided $(n=54)$ & P-value & Right-sided $(n=28)$ & Left-sided $(n=28)$ & P-value \\
\hline Age (yr) & $67(28-95)$ & $71(38-91)$ & 0.086 & $71(32-95)$ & $70(38-90)$ & 0.646 \\
\hline Sex & & & 0.031 & & & 0.789 \\
\hline Male & 27 (48.2) & $37(68.5)$ & & $14(50.0)$ & $15(53.6)$ & \\
\hline Female & $29(51.8)$ & $17(31.5)$ & & $14(50.0)$ & $13(46.4)$ & \\
\hline Adjuvant chemotherapy & & & 0.432 & & & 0.383 \\
\hline Absent & $45(80.4)$ & $40(74.1)$ & & $18(64.3)$ & $21(75.0)$ & \\
\hline Present & $11(19.6)$ & $14(25.9)$ & & $10(35.7)$ & $7(25.0)$ & \\
\hline T factor, UICC 7th ed & & & 0.031 & & & 0.752 \\
\hline $\mathrm{T} 1-3$ & 48 (85.7) & $37(68.5)$ & & $22(78.6)$ & $21(75.0)$ & \\
\hline $\mathrm{T} 4$ & $8(14.3)$ & $17(31.5)$ & & $6(21.4)$ & $7(25.0)$ & \\
\hline Lymphatic invasion & & & 0.146 & & & $>0.999$ \\
\hline ly0-1 & 46 (82.1) & $38(70.4)$ & & $24(85.7)$ & $24(85.7)$ & \\
\hline ly2-3 & $10(17.9)$ & $16(29.6)$ & & $4(14.3)$ & 4 (14.3) & \\
\hline Venous invasion & & & 0.002 & & & $>0.999$ \\
\hline v0-1 & 48 (85.7) & $32(59.3)$ & & $26(92.9)$ & $25(89.3)$ & \\
\hline v2-3 & $8(14.3)$ & $22(40.7)$ & & $2(7.1)$ & $3(10.7)$ & \\
\hline \multicolumn{2}{|c|}{ Histological differentiation of primary colorectal cancer } & & 0.003 & & & 0.459 \\
\hline Pap & $7(12.5)$ & $1(1.9)$ & & $4(14.3)$ & $1(3.6)$ & \\
\hline Tub1 & $12(21.4)$ & $27(50.0)$ & & $9(32.1)$ & $11(39.3)$ & \\
\hline Tub2 & $30(53.6)$ & $24(44.4)$ & & $14(50.0)$ & $14(50.0)$ & \\
\hline Muc/por & $7(12.5)$ & $2(3.7)$ & & $1(3.6)$ & $2(7.1)$ & \\
\hline CEA (ng/mL) & & & 0.941 & & & 0.554 \\
\hline$\geq 10$ & $18(32.1)$ & $17(31.5)$ & & $9(32.1)$ & $7(25.0)$ & \\
\hline$<10$ & $38(67.9)$ & $37(68.5)$ & & 19 (67.9) & $21(75.0)$ & \\
\hline CA19-9 (U/mL) & & & 0.862 & & & $>0.999$ \\
\hline$\geq 37$ & $10(17.9)$ & $8(14.8)$ & & $4(14.3)$ & $4(14.3)$ & \\
\hline$<37$ & $46(82.1)$ & $46(85.2)$ & & $24(85.7)$ & $24(85.7)$ & \\
\hline Lymph node cleaning & & & 0.089 & & & 0.252 \\
\hline D3 & $51(91.1)$ & $43(79.6)$ & & $26(92.9)$ & $22(78.6)$ & \\
\hline D1-2 & $5(8.9)$ & $11(20.4)$ & & $2(7.1)$ & $6(21.4)$ & \\
\hline Laparoscopic approach & & & 0.533 & & & 0.786 \\
\hline Present & $24(42.9)$ & $20(27.0)$ & & $12(42.9)$ & $11(39.3)$ & \\
\hline Absent & $32(57.1)$ & $34(63.0)$ & & $16(57.1)$ & $17(60.7)$ & \\
\hline Estimated blood loss (mL) & & & 0.154 & & & 0.252 \\
\hline$\geq 200$ & $11(19.6)$ & $17(31.5)$ & & $7(25.0)$ & $11(39.3)$ & \\
\hline$<200$ & $45(80.4)$ & $37(68.5)$ & & $21(75.0)$ & $17(60.7)$ & \\
\hline Operative duration (min) & & & 0.835 & & & 0.179 \\
\hline$\geq 240$ & $30(53.6)$ & $30(55.6)$ & & $13(46.4)$ & $18(64.3)$ & \\
\hline$<240$ & $26(46.4)$ & $24(44.4)$ & & $15(53.6)$ & $10(35.7)$ & \\
\hline
\end{tabular}

Values are presented as median (range) or number (\%).

UICC, Union for International Cancer Control; pap, papillary adenocarcinoma; tub1, well-differentiated tubular adenocarcinoma; tub2, moderately differentiated tubular adenocarcinoma; muc, mucinous adenocarcinoma; por, poorly differentiated adenocarcinoma; CEA, carcinoembryonic antigen; CA19-9, carbohydrate antigen 19-9. 


\section{RESULTS}

\section{Characteristics and outcomes of stage II colon cancer}

Table 1 shows the characteristics of the patients with stage II colon cancer. There were 56 patients in the RSC group and 54 patients in the LSC group. The proportion of women was significantly higher in the RSC group ( $51.8 \%$ vs. $31.5 \%, \mathrm{P}=0.031$ ). The proportion of tumor stage T4 was significantly higher in the LSC group $31.5 \%$ vs. $14.3 \%, \mathrm{P}=0.031$ ). The proportion of $\mathrm{v} 2-3$ was significantly higher in the LSC group ( $40.7 \%$ vs. $14.3 \%, \mathrm{P}=0.002)$. The proportion of mucinous or poorly differentiated adenocarcinoma was

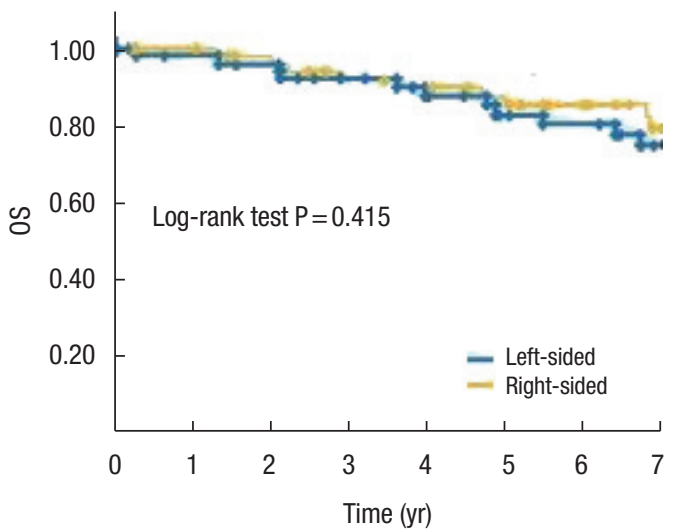

\begin{tabular}{lllllllll}
\hline Number at risk & & & & & & & & \\
\hline Left-sided & 65 & 64 & 60 & 49 & 35 & 31 & 28 & 23 \\
Right-sided & 41 & 40 & 33 & 27 & 17 & 13 & 10 & 10 \\
\hline
\end{tabular}

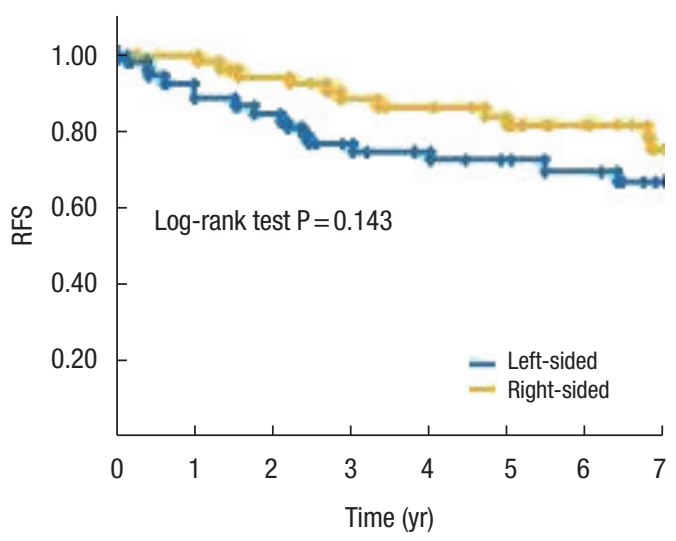

\begin{tabular}{lllllllll}
\hline Number at risk & \multicolumn{1}{l}{ N } & & & & & & & \\
\hline Left-sided & 54 & 48 & 44 & 36 & 31 & 29 & 27 & 22 \\
Right-sided & 56 & 54 & 49 & 43 & 41 & 37 & 30 & 23 \\
\hline
\end{tabular}

significantly higher in the RSC group (12.5\% vs. $3.7 \%, \mathrm{P}=0.003$ ). After propensity matching, 28 pairs were extracted. There was no significant difference between the RSC and the LSC groups. Fig. 1A and B show the OS and the RFS of the patients with stage II colon cancer before propensity matching. No significant difference was observed. Fig. 1C and D show the results after propensity matching. There was no significant difference.

\section{Characteristics and outcomes of stage III colon cancer}

Table 2 shows the characteristics of the patients with stage III colon cancer. There were 48 patients in the RSC group and 52 patients

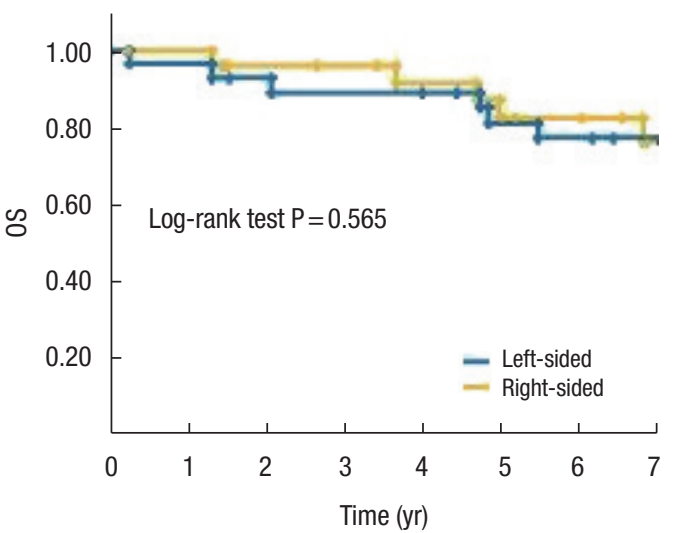

\begin{tabular}{lllllllll}
\hline Number at risk & & & & & & & & \\
\hline Left-sided & 28 & 28 & 26 & 26 & 25 & 24 & 21 & 20 \\
Right-sided & 28 & 27 & 24 & 23 & 21 & 19 & 16 & 12 \\
\hline
\end{tabular}

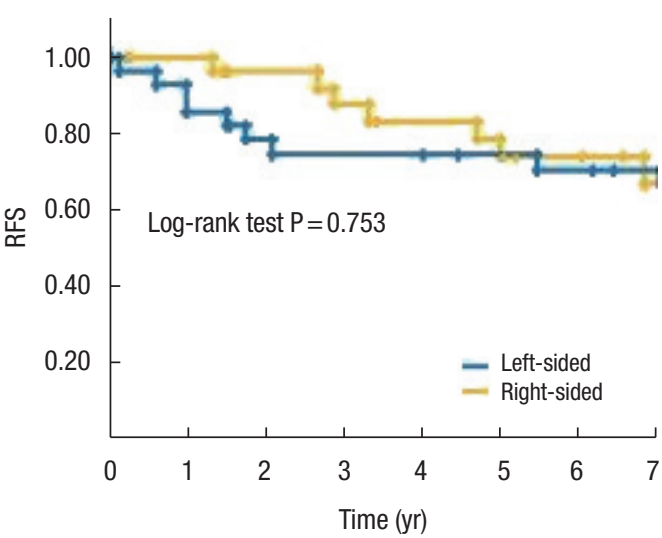

\begin{tabular}{lllllllll}
\hline Number at risk & 110 & 18 & 16 & \\
\hline Left-sided & 28 & 26 & 22 & 21 & 20 & 19 & 18 & 16 \\
Right-sided & 28 & 27 & 24 & 21 & 19 & 17 & 14 & 10
\end{tabular}

Fig. 1. Kaplan-Meier curve of overall survival (OS) and recurrence-free survival (RFS) for patients with stage II colon cancer before propensity score matching $(\mathrm{A}, \mathrm{B})$ and after propensity score matching $(\mathrm{C}, \mathrm{D})$. (A) OS did not differ significantly between the groups $(\mathrm{P}=0.415)$. (B) RFS did not differ significantly between the groups $(\mathrm{P}=0.143)$. (C) OS did not differ significantly between the groups $(\mathrm{P}=0.565)$. (D) RFS did not differ significantly between the groups $(\mathrm{P}=0.753)$. 
Table 2. Characteristics of the patients with stage III colon cancer

\begin{tabular}{|c|c|c|c|c|c|c|}
\hline \multirow{2}{*}{ Variable } & \multicolumn{3}{|c|}{ Before propensity matching } & \multicolumn{3}{|c|}{ After propensity matching } \\
\hline & Right-sided $(n=48)$ & Left-sided $(n=52)$ & P-value & Right-sided $(n=32)$ & Left-sided $(n=32)$ & P-value \\
\hline Age (yr) & $73(45-90)$ & 68 (43-92) & 0.005 & $72(45-87)$ & $72(54-86)$ & 0.185 \\
\hline Sex & & & 0.652 & & & 0.211 \\
\hline Male & $20(41.7)$ & 24 (46.2) & & $13(40.6)$ & 18 (56.3) & \\
\hline Female & $28(58.3)$ & $28(53.8)$ & & $19(59.4)$ & $14(43.8)$ & \\
\hline Adjuvant chemotherapy & & & 0.921 & & & 0.302 \\
\hline Absent & $18(37.5)$ & $19(36.5)$ & & $14(43.8)$ & 10 (31.3) & \\
\hline Present & $30(62.5)$ & $33(63.5)$ & & 18 (56.3) & $22(68.8)$ & \\
\hline T factor, UICC 7th ed & & & 0.016 & & & $>0.999$ \\
\hline $\mathrm{T} 1-3$ & 40 (83.3) & $32(61.5)$ & & $24(75.0)$ & $24(75.0)$ & \\
\hline T4 & $8(16.7)$ & $20(38.5)$ & & $8(25.0)$ & $8(25.0)$ & \\
\hline \multicolumn{2}{|c|}{$\mathrm{N}$ factor of primary colorectal cancer, UICC 7th ed } & & 0.159 & & & 0.708 \\
\hline No-1 & $34(70.8)$ & $43(82.7)$ & & $29(90.6)$ & $27(84.4)$ & \\
\hline N2-3 & $14(29.2)$ & $9(17.3)$ & & $3(9.4)$ & $5(15.6)$ & \\
\hline Lymphatic invasion & & & 0.466 & & & 0.790 \\
\hline ly0-1 & $32(66.7)$ & $31(59.6)$ & & $22(68.8)$ & $21(65.6)$ & \\
\hline ly2-3 & 16 (33.3) & $21(40.4)$ & & $10(31.3)$ & $11(34.4)$ & \\
\hline Venous invasion & & & 0.127 & & & $>0.999$ \\
\hline v0-1 & $33(68.8)$ & $28(53.8)$ & & $20(62.5)$ & $20(62.5)$ & \\
\hline v2-3 & $15(31.2)$ & $24(46.2)$ & & $12(37.5)$ & $12(37.5)$ & \\
\hline \multicolumn{2}{|c|}{ Histological differentiation of primary colorectal cancer } & & 0.074 & & & 0.466 \\
\hline Pap & $1(2.1)$ & $3(5.8)$ & & $1(3.1)$ & 0 & \\
\hline Tub1 & $20(41.7)$ & $11(21.2)$ & & $10(31.2)$ & $11(34.4)$ & \\
\hline Tub2 & $22(45.8)$ & $35(67.3)$ & & $19(59.4)$ & $19(59.4)$ & \\
\hline Muc/por & $5(10.4)$ & $3(5.8)$ & & $2(6.3)$ & $2(6.3)$ & \\
\hline CEA (ng/mL) & & & 0.254 & & & 0.281 \\
\hline$\geq 10$ & $16(33.3)$ & $12(23.1)$ & & $12(37.5)$ & $8(25.0)$ & \\
\hline$<10$ & $32(66.7)$ & $40(76.9)$ & & $20(62.5)$ & $24(75.0)$ & \\
\hline CA19-9 (U/mL) & & & 0.118 & & & 0.302 \\
\hline$\geq 37$ & $10(20.8)$ & $5(9.6)$ & & $7(21.9)$ & $3(9.4)$ & \\
\hline$<37$ & $38(79.2)$ & $47(90.4)$ & & $25(78.1)$ & $29(90.6)$ & \\
\hline Lymph node cleaning & & & 0.313 & & & 0.355 \\
\hline D3 & 39 (81.3) & $46(88.5)$ & & $28(87.5)$ & 31 (96.9) & \\
\hline D1-2 & $9(18.8)$ & $6(11.5)$ & & 4 (12.5) & $1(3.1)$ & \\
\hline Laparoscopic approach & & & 0.005 & & & 0.024 \\
\hline Present & $16(33.3)$ & $32(61.5)$ & & $11(34.4)$ & 20 (62.5) & \\
\hline Absent & $32(66.7)$ & $20(38.5)$ & & $21(65.6)$ & $12(37.5)$ & \\
\hline Estimated blood loss (mL) & & & 0.313 & & & 0.777 \\
\hline$\geq 200$ & $9(18.8)$ & 10 (19.2) & & $8(25.0)$ & $9(28.1)$ & \\
\hline$<200$ & 39 (81.3) & $42(80.8)$ & & $24(75.0)$ & $23(71.9)$ & \\
\hline Operative duration (min) & & & 0.951 & & & $>0.999$ \\
\hline$\geq 240$ & $20(41.7)$ & $25(48.1)$ & & $14(43.8)$ & $14(43.8)$ & \\
\hline$<240$ & $28(58.3)$ & 27 (51.9) & & $18(56.3)$ & $18(56.3)$ & \\
\hline
\end{tabular}

Values are presented as median (range) or number (\%).

UICC, Union for International Cancer Control; pap, papillary adenocarcinoma; tub1, well-differentiated tubular adenocarcinoma; tub2, moderately differentiated tubular adenocarcinoma; muc, mucinous adenocarcinoma; por, poorly differentiated adenocarcinoma; CEA, carcinoembryonic antigen; CA19-9, carbohydrate antigen 19-9. 


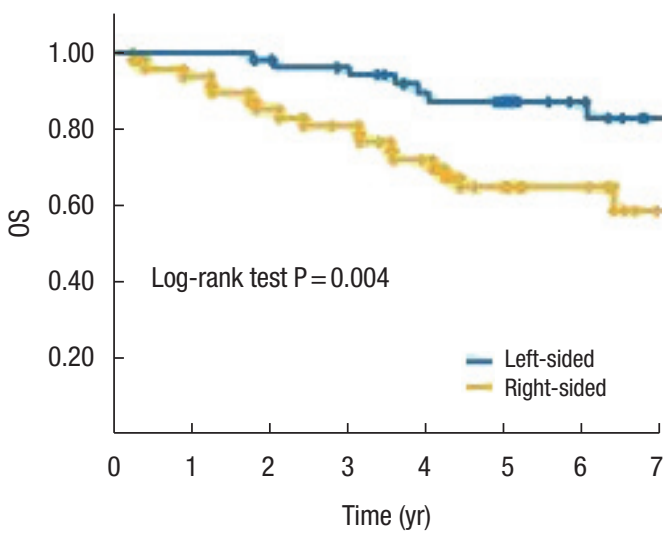

\begin{tabular}{llllllllr}
\hline Number at risk & & & & & & & & \\
\hline Left-sided & 48 & 45 & 41 & 38 & 32 & 22 & 15 & 8 \\
Right-sided & 52 & 52 & 49 & 46 & 40 & 34 & 22 & 16 \\
\hline
\end{tabular}

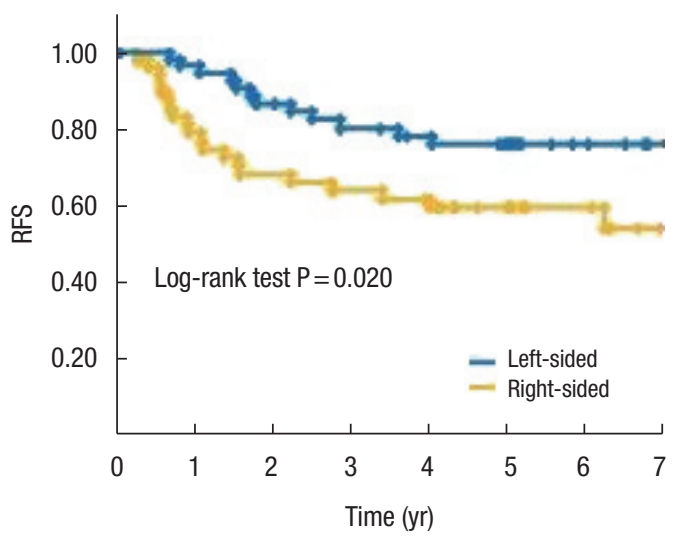

\begin{tabular}{llllllllr}
\hline Number at risk & \multicolumn{1}{l}{ N } & & & & & & & \\
\hline Left-sided & 48 & 38 & 33 & 30 & 26 & 19 & 13 & 7 \\
Right-sided & 52 & 50 & 42 & 39 & 35 & 30 & 18 & 14 \\
\hline
\end{tabular}

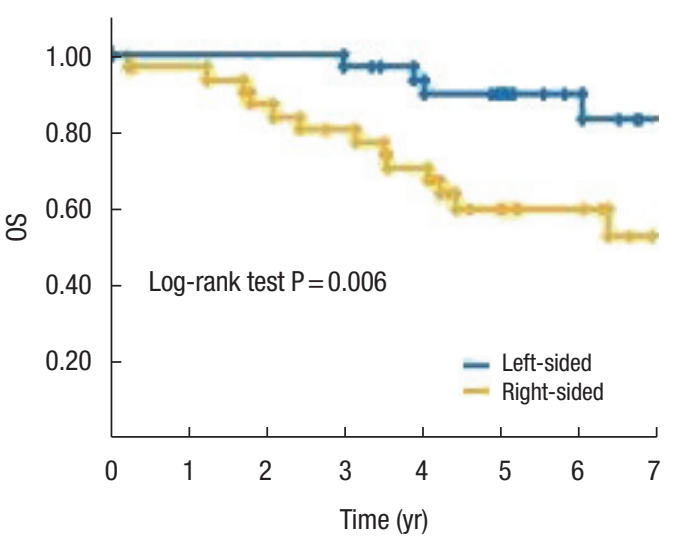

\begin{tabular}{lllllllll}
\hline Number at risk & & & & & & & & \\
\hline Left-sided & 32 & 32 & 32 & 31 & 28 & 23 & 15 & 10 \\
Right-sided & 32 & 31 & 28 & 25 & 22 & 15 & 11 & 6 \\
\hline
\end{tabular}

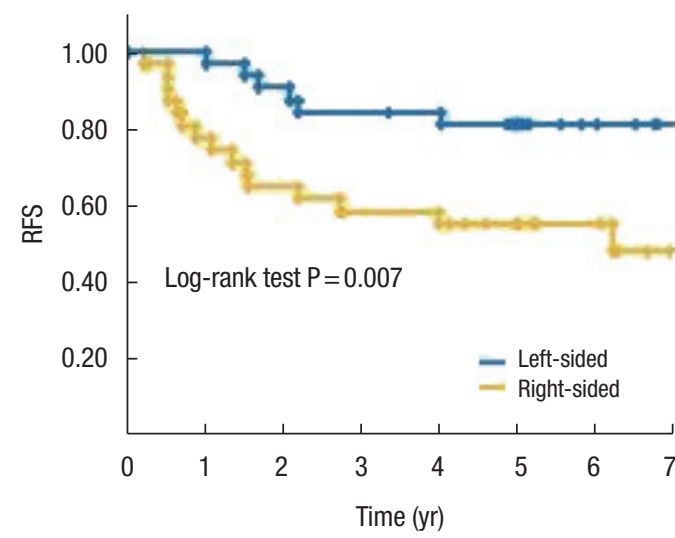

\begin{tabular}{lllllllll}
\hline Number at risk & 10 & & & & & & & \\
\hline Left-sided & 32 & 32 & 30 & 28 & 26 & 21 & 13 & 9 \\
Right-sided & 32 & 25 & 21 & 18 & 17 & 13 & 10 & 5 \\
\hline
\end{tabular}

Fig. 2. Kaplan-Meier curve of overall survival (OS) and recurrence-free survival (RFS) for patients with stage III colon cancer before propensity score matching $(A, B)$ and after propensity score matching $(C, D)$. (A) OS of the right-sided group was significantly worse than the leftsided group $(\mathrm{P}=0.004)$. (B) RFS of the right-sided group was significantly worse than the left-sided group $(\mathrm{P}=0.020)$. (C) OS of the rightsided group was significantly worse than the left-sided group $(\mathrm{P}=0.004)$. (D) RFS of the right-sided group was significantly worse than the left-sided group $(\mathrm{P}=0.020)$.

in the LSC group. The median age was significantly older in the RSC group ( 73 vs. 68 years, $P=0.005$ ). The proportion of tumor stage T4 was significantly higher in the LSC group ( 38.5 vs. $16.7 \%$, $\mathrm{P}=0.016$ ). After propensity matching, 32 pairs were extracted. There was no significant difference between the RSC and LSC groups. Fig. 2A and B show the OS and RFS of the patients with stage III colon cancer before propensity matching. The OS and RFS of the RSC group were both significantly worse than the LSC group $(\mathrm{P}=0.004$ and $\mathrm{P}=0.020$, respectively). Fig. $2 \mathrm{C}$ and $\mathrm{D}$ show the results after propensity matching. The OS and the RFS of RSC group were both significantly worse than the LSC group $(\mathrm{P}=0.006$ and $\mathrm{P}=0.007$, respectively).

\section{Univariate and multivariate analysis of prognostic factors for stage II colon cancer}

Table 3 shows the analysis of prognostic factors for OS in stage II colon cancer. Univariate analysis showed no prognostic impact in location of the colon cancer. Laparoscopic approach, estimated blood loss of $\geq 200 \mathrm{~mL}$, and operative duration of $<240$ minutes showed significant difference in univariate analysis, while multivariate analysis showed no significant prognostic factors. 
Table 3. Uni- and multivariate analysis of overall survival for the stage II colon cancer

\begin{tabular}{|c|c|c|c|c|c|c|c|c|}
\hline \multirow{2}{*}{ Variable } & \multirow{2}{*}{ Category } & \multirow{2}{*}{$\begin{array}{c}\text { No. of } \\
\text { patients }\end{array}$} & \multicolumn{3}{|c|}{ Univariate analysis } & \multicolumn{3}{|c|}{ Multivariate analysis } \\
\hline & & & $\mathrm{HR}$ & $95 \% \mathrm{Cl}$ & P-value & $\mathrm{HR}$ & $95 \% \mathrm{Cl}$ & P-value \\
\hline Age (yr) & $\geq 70 /<70$ & $66 / 44$ & 1.714 & $0.733-4.010$ & 0.214 & - & - & - \\
\hline Sex & Male/female & $64 / 46$ & 0.825 & $0.370-1.838$ & 0.638 & - & - & - \\
\hline Adjuvant chemotherapy & Absent/present & $85 / 25$ & 0.735 & $0.251-2.152$ & 0.575 & - & - & - \\
\hline T factor, UICC 7th ed & $\mathrm{T} 4 / \mathrm{T} 1-3$ & $25 / 85$ & 0.701 & $0.239-2.052$ & 0.516 & - & - & - \\
\hline Lymphatic invasion & ly2-3/ly0-1 & $26 / 84$ & 0.511 & $0.175-1.491$ & 0.219 & - & - & - \\
\hline Venous invasion & v2-3/v0-1 & $30 / 80$ & 0.567 & $0.213-1.511$ & 0.257 & - & - & - \\
\hline Histological differentiation & Muc/pap/others & $9 / 101$ & 1.395 & $0.325-5.978$ & 0.654 & - & - & - \\
\hline CEA (ng/mL) & $\geq 10 /<10$ & $34 / 76$ & 1.775 & $0.804-3.919$ & 0.156 & - & - & - \\
\hline CA19-9 (U/mL) & $\geq 37 /<37$ & $18 / 92$ & 1.257 & $0.465-3.398$ & 0.652 & - & - & - \\
\hline Lymph node cleaning & D1-2/D3 & $16 / 94$ & 0.890 & $0.265-2.990$ & 0.850 & - & - & - \\
\hline Laparoscopic approach & Present/absent & $44 / 66$ & 3.922 & $1.329-11.573$ & 0.013 & 1.851 & $0.509-6.724$ & 0.350 \\
\hline Estimated blood loss (mL) & $\geq 200 /<200$ & $28 / 82$ & 2.543 & $1.138-5.684$ & 0.023 & 2.425 & $0.932-6.308$ & 0.075 \\
\hline Operative duration (min) & $\geq 240 /<240$ & $60 / 50$ & 0.418 & $0.184-0.951$ & 0.037 & 0.424 & $0.165-1.089$ & 0.069 \\
\hline Location of the colon cancer & Right/left & $56 / 54$ & 0.718 & $0.325-1.590$ & 0.415 & & & \\
\hline
\end{tabular}

HR, hazard ratio; Cl, confidence interval; UICC, Union for International Cancer Control; muc, mucinous adenocarcinoma; pap, papillary adenocarcinoma; CEA, carcinoembryonic antigen; CA19-9, carbohydrate antigen 19-9.

Table 4. Uni- and multivariate analysis of overall survival for the stage III colon cancer

\begin{tabular}{|c|c|c|c|c|c|c|c|c|}
\hline \multirow{2}{*}{ Variable } & \multirow{2}{*}{ Category } & \multirow{2}{*}{$\begin{array}{c}\text { No. of } \\
\text { patients }\end{array}$} & \multicolumn{3}{|c|}{ Univariate analysis } & \multicolumn{3}{|c|}{ Multivariate analysis } \\
\hline & & & $\mathrm{HR}$ & $95 \% \mathrm{Cl}$ & P-value & $\mathrm{HR}$ & $95 \% \mathrm{Cl}$ & P-value \\
\hline Age (yr) & $\geq 70 /<70$ & $64 / 36$ & 1.640 & $0.693-3.881$ & 0.261 & - & - & - \\
\hline Sex & Male/female & $44 / 56$ & 0.662 & $0.303-1.448$ & 0.302 & - & - & - \\
\hline Adjuvant chemotherapy & Absent/present & $37 / 63$ & 0.544 & $0.255-1.163$ & 0.116 & - & - & - \\
\hline T factor, UICC 7th ed & $\mathrm{T} 4 / \mathrm{T} 1-3$ & $23 / 77$ & 0.572 & $0.228-1.436$ & 0.234 & - & - & - \\
\hline $\mathrm{N}$ factor, UICC 7th ed & N2-3/N1 & $23 / 77$ & 0.678 & $0.231-1.984$ & 0.478 & - & - & - \\
\hline Lymphatic invasion & ly2-3/ly0-1 & $37 / 63$ & 0.551 & $0.232-1.309$ & 0.177 & - & - & - \\
\hline Venous invasion & $\mathrm{v} 2-3 / v 0-1$ & $39 / 61$ & 1.096 & $0.511-2.347$ & 0.814 & - & - & - \\
\hline Histological differentiation & Muc/pap/other & $8 / 92$ & 0.530 & $0.071-3.928$ & 0.534 & - & - & - \\
\hline CEA (ng/mL) & $\geq 10 /<10$ & $28 / 72$ & 2.350 & $1.093-5.054$ & 0.029 & 2.699 & $1.196-6.088$ & 0.017 \\
\hline CA19-9 (U/mL) & $\geq 37 /<37$ & $15 / 85$ & 1.548 & $0.583-4.111$ & 0.380 & - & - & - \\
\hline Lymph node cleaning & D1-2/D3 & $15 / 85$ & 0.408 & $0.172-0.967$ & 0.042 & 0.452 & $0.138-1.016$ & 0.105 \\
\hline Laparoscopic approach & Present/absent & $48 / 52$ & 1.937 & $0.869-4.316$ & 0.106 & - & - & - \\
\hline Estimated blood loss (mL) & $\geq 200 /<200$ & $19 / 81$ & 0.822 & $0.310-2.183$ & 0.695 & - & - & - \\
\hline Operative duration (min) & $\geq 240 /<240$ & $45 / 55$ & 0.665 & $0.304-1.452$ & 0.306 & - & - & - \\
\hline Location of the colon cancer & Right/left & $48 / 52$ & 3.145 & 1.372-7.194 & 0.007 & 2.688 & $1.786-6.211$ & 0.021 \\
\hline
\end{tabular}

HR, hazard ratio; Cl, confidence interval; UICC, Union for International Cancer Control; muc, mucinous adenocarcinoma; pap, papillary adenocarcinoma; CEA, carcinoembryonic antigen; CA19-9, carbohydrate antigen 19-9.

\section{Univariate and multivariate analysis of prognostic factors for stage III colon cancer}

Table 4 shows the analysis of prognostic factors for OS in stage III colon cancer. Univariate analysis revealed RSC was a poor prog- nostic factor. CEA of $\geq 10 \mathrm{ng} / \mathrm{mL}$ and D3 lymph node cleaning were also identified as poor prognostic factors. Multivariate analysis revealed RSC (hazard ratio $[\mathrm{HR}], 2.688 ; \mathrm{P}=0.007$ ) and CEA of $\geq 10 \mathrm{ng} / \mathrm{mL}(\mathrm{HR}, 2.699 ; \mathrm{P}=0.017)$ were independent poor prog- 
nostic factors for OS in stage III colon cancer.

\section{Characteristics and outcomes of liver metastasis}

Table 5 shows the characteristics of the patients with liver metastasis. There were 41 patients in the RSC group and 65 patients in the LSC group. The proportion of synchronous metastasis, number of liver metastases, and maximum tumor size were similar in the RSC and LSC groups. The proportions of tumor stages T4,

Table 5. Characteristics of the patients with liver metastasis of colon cancer

\begin{tabular}{|c|c|c|c|}
\hline Variable & $\begin{array}{l}\text { Right-sided } \\
\quad(\mathrm{n}=41)\end{array}$ & $\begin{array}{l}\text { Left-sided } \\
\qquad(n=65)\end{array}$ & P-value \\
\hline Age (yr) & $68(43-84)$ & $65(38-84)$ & 0.203 \\
\hline \multicolumn{3}{|l|}{ Sex } & 0.261 \\
\hline Male & $24(58.5)$ & $45(69.2)$ & \\
\hline Female & $17(41.5)$ & $20(30.8)$ & \\
\hline \multicolumn{3}{|l|}{ Timing of liver metastasis } & 0.202 \\
\hline Synchronous & $15(36.6)$ & $32(49.2)$ & \\
\hline Metachronous & $26(63.4)$ & $33(50.8)$ & \\
\hline \multicolumn{3}{|l|}{ No. of liver metastasis } & 0.182 \\
\hline 1 & $21(51.2)$ & $29(44.6)$ & \\
\hline $2-4$ & $15(36.6)$ & $23(35.4)$ & \\
\hline$\geq 5$ & $5(12.2)$ & $13(20.0)$ & \\
\hline \multicolumn{3}{|l|}{ Maximum tumor size } & 0.297 \\
\hline$\geq 5$ & $5(12.2)$ & $13(20.0)$ & \\
\hline$<5$ & $36(87.8)$ & $52(80.0)$ & \\
\hline \multicolumn{3}{|c|}{ T factor of primary colorectal cancer, UICC 7th ed } & 0.593 \\
\hline $\mathrm{T} 1-3$ & $31(75.6)$ & $52(80.0)$ & \\
\hline $\mathrm{T} 4$ & $10(24.4)$ & $13(20.0)$ & \\
\hline \multicolumn{3}{|c|}{$\mathrm{N}$ factor of primary colorectal cancer, UICC 7th ed } & 0.707 \\
\hline No-1 & $31(75.6)$ & $47(72.3)$ & \\
\hline N2-3 & $10(24.4)$ & $18(27.7)$ & \\
\hline \multicolumn{3}{|c|}{ Lymphatic invasion of primary colorectal cancer } & 0.880 \\
\hline ly0-1 & $28(68.3)$ & $47(72.3)$ & \\
\hline ly2-3 & $13(31.7)$ & $18(27.7)$ & \\
\hline \multicolumn{3}{|c|}{ Venous invasion of primary colorectal cancer } & 0.627 \\
\hline v0-1 & $22(53.7)$ & $38(58.5)$ & \\
\hline v2-3 & $19(46.3)$ & $27(41.5)$ & \\
\hline \multicolumn{3}{|c|}{ Histological differentiation of primary colorectal cancer } & 0.485 \\
\hline Pap & $0(0)$ & $1(1.5)$ & \\
\hline Tub1 & $15(36.6)$ & $21(32.3)$ & \\
\hline Tub2 & $22(53.7)$ & $39(60.0)$ & \\
\hline Muc/por & $4(9.8)$ & $4(6.2)$ & \\
\hline
\end{tabular}

(Continued to the next)
$\mathrm{N} 2-3$, ly2 -3 , and $\mathrm{v} 2-3$ of the primary colon cancer were similar in the 2 groups. The proportion of patients whose CA19-9 level was $>37 \mathrm{U} / \mathrm{mL}$ was significantly higher in the RSC group $(56.1 \%$ vs. $32.3 \%, P=0.016)$. Fig. $3 \mathrm{~A}$ and $\mathrm{B}$ show the OS and RFS after liver resection for the patients with liver metastasis. The OS of the RSC group was significantly worse than the LSC group $(\mathrm{P}=0.032$; Fig. 3A), while no significant difference was observed in RFS ( $\mathrm{P}=$ 0.412; Fig. 3B).

\section{Outcomes of metachronous liver metastasis}

Fig. 3C shows the OS after liver resection for the patients with metachronous liver metastasis whose primary colon cancer was stage II. There was no significant difference between the RSC and LSC groups $(\mathrm{P}=0.668)$. Fig. $3 \mathrm{D}$ shows the $\mathrm{OS}$ after liver resection for the patients with metachronous liver metastasis whose primary colon cancer was stage III. The OS of the RSC group was significantly worse than the LSC group $(\mathrm{P}=0.026)$.

\section{Details of the recurrent site after curative resection}

Table 6 summarizes the details of site of recurrence by each cancer stage. The recurrent sites include initial recurrence and second and subsequent recurrent sites. The proportion of peritoneal dissemination was significantly higher in the RSC group compared with the LSC group in those with stage II (80.0\% vs. $15.4 \%, \mathrm{P}=$ $0.022)$, stage III ( $62.5 \%$ vs. $27.3 \%, \mathrm{P}=0.022)$, and liver metastasis (23.3\% vs. $6.4 \%, \mathrm{P}=0.041)$.

Table 5. Continued

\begin{tabular}{lccc}
\hline Variable & $\begin{array}{c}\text { Right-sided } \\
(\mathrm{n}=41)\end{array}$ & $\begin{array}{c}\text { Left-sided } \\
(\mathrm{n}=65)\end{array}$ & P-value \\
\hline CEA $(\mathrm{ng} / \mathrm{mL})$ & & & 0.862 \\
$\quad \geq 10$ & $22(53.7)$ & $36(55.4)$ & \\
$\quad<10$ & $19(46.3)$ & $29(44.6)$ & \\
CA19-9 (U/mL) & & & 0.016 \\
$\geq 37$ & $23(56.1)$ & $21(32.3)$ & \\
$\quad<37$ & $18(43.9)$ & $44(67.7)$ & \\
Laparoscopic approach & & & 0.658 \\
$\quad$ Present & $13(31.7)$ & $18(27.7)$ & \\
Absent & $28(68.3)$ & $47(72.3)$ & \\
Adjuvant chemotherapy & & & 0.535 \\
Present & $26(63.4)$ & $45(69.2)$ & \\
Absent & $15(36.6)$ & $20(30.8)$ & \\
\hline
\end{tabular}

Values are presented as median (range) or number (\%).

UICC, Union for International Cancer Control; pap, papillary adenocarcinoma; tub1, well-differentiated tubular adenocarcinoma; tub2, moderately differentiated tubular adenocarcinoma; muc, mucinous adenocarcinoma; por, poorly differentiated adenocarcinoma; CEA, carcinoembryonic antigen; CA19-9, carbohydrate antigen 19-9. 


\section{Coloproctologogy}

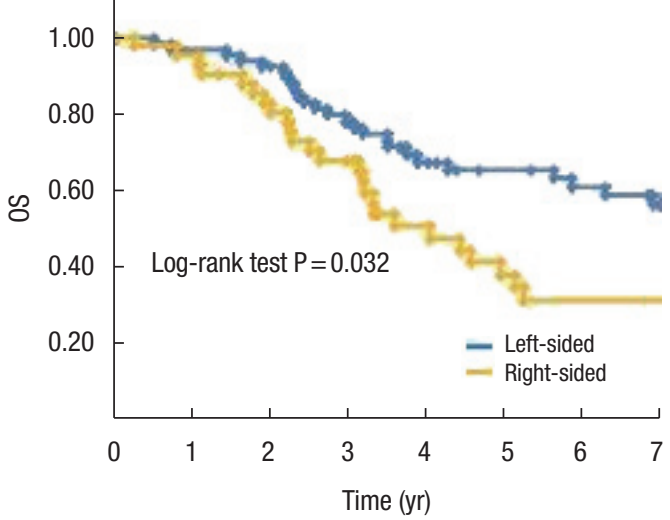

\begin{tabular}{|c|c|c|c|c|c|c|c|c|}
\hline \multicolumn{9}{|c|}{ Number at risk } \\
\hline Left-sided & 65 & 64 & 60 & 49 & 35 & 31 & 28 & 23 \\
\hline Right-sided & 41 & 40 & 33 & 27 & 17 & 13 & 10 & 10 \\
\hline
\end{tabular}

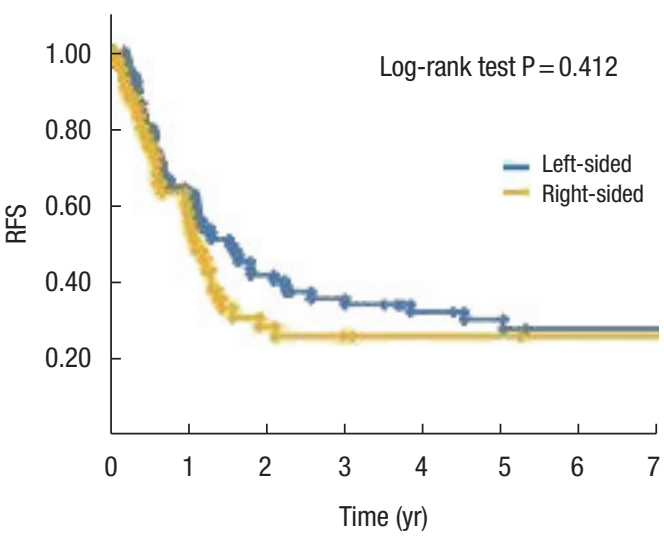

\begin{tabular}{|c|c|c|c|c|c|c|c|c|}
\hline \multicolumn{9}{|c|}{ Number at risk } \\
\hline \multicolumn{9}{|c|}{$\frac{\text { Number at risk }}{\text { Left-sided }}$} \\
\hline Right-sided & 41 & 22 & 12 & 10 & 9 & 9 & 8 & 8 \\
\hline
\end{tabular}

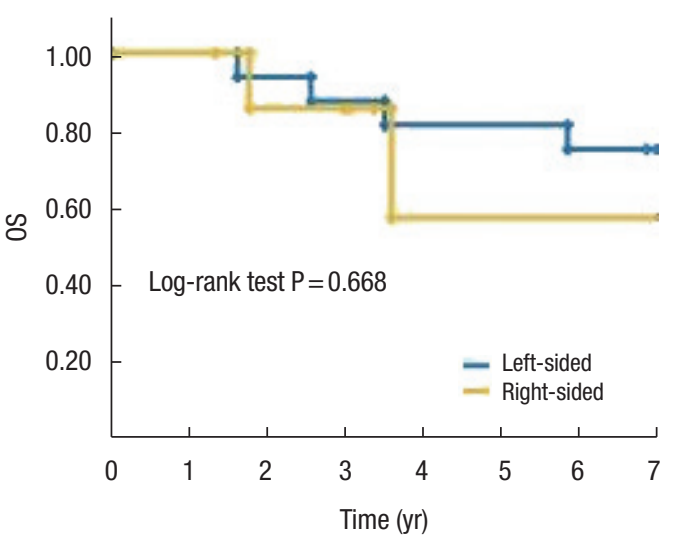

\begin{tabular}{lrrrrrrrr}
\hline Number at risk & & & & & & & & \\
\hline Left-sided & 16 & 16 & 15 & 15 & 14 & 13 & 12 & 11 \\
Right-sided & 8 & 8 & 7 & 6 & 3 & 3 & 3 & 3 \\
\hline
\end{tabular}

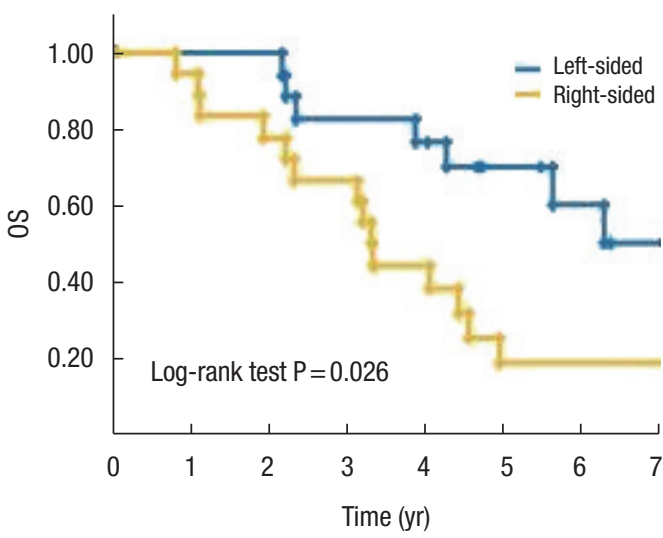

\begin{tabular}{|c|c|c|c|c|c|c|c|c|}
\hline \multicolumn{9}{|c|}{ Number at risk } \\
\hline Left-sided & 17 & 17 & 17 & 14 & 13 & 9 & 7 & 5 \\
\hline Right-sided & 18 & 17 & 15 & 13 & 8 & 4 & 4 & 4 \\
\hline
\end{tabular}

Fig. 3. Kaplan-Meier curve of overall survival (OS) and recurrence-free survival (RFS) for patients with liver metastasis. (A) OS of the rightsided group was significantly worse than the left-sided group $(\mathrm{P}=0.032)$. (B) RFS did not differ significantly between the groups $(\mathrm{P}=0.412)$. (C) Result of the metachronous liver metastasis whose primary colon cancer was stage II. OS did not differ significantly between the groups $(\mathrm{P}=0.668)$. (D) Result of the metachronous liver metastasis whose primary colon cancer was stage III. The right-sided group was significantly worse than the left-sided group $(\mathrm{P}=0.026)$.

Table 6. Recurrent site after curative resection

\begin{tabular}{|c|c|c|c|c|c|c|c|c|c|}
\hline \multirow[b]{2}{*}{ Variable } & \multicolumn{3}{|c|}{ Stage II } & \multicolumn{3}{|c|}{ Stage III } & \multicolumn{3}{|c|}{ Liver metastasis } \\
\hline & $\begin{array}{l}\text { Right-sided } \\
\quad(\mathrm{n}=5)\end{array}$ & $\begin{array}{l}\text { Left-sided } \\
(n=13)\end{array}$ & P-value & $\begin{array}{l}\text { Right-sided } \\
\quad(n=16)\end{array}$ & $\begin{array}{l}\text { Left-sided } \\
(n=11)\end{array}$ & P-value & $\begin{array}{l}\text { Right-sided } \\
(\mathrm{n}=30)\end{array}$ & $\begin{array}{l}\text { Left-sided } \\
(n=47)\end{array}$ & P-value \\
\hline Liver & $1(20.0)$ & $8(61.5)$ & 0.294 & $7(43.8)$ & $5(45.5)$ & 0.759 & $20(66.7)$ & $37(78.7)$ & 0.239 \\
\hline Lung & $2(40.0)$ & $5(38.5)$ & $>0.999$ & $4(25.0)$ & $3(27.3)$ & $>0.999$ & $4(13.3)$ & $10(21.3)$ & 0.547 \\
\hline Peritoneal dissemination & $4(80.0)$ & $2(15.4)$ & 0.022 & $10(62.5)$ & $3(27.3)$ & 0.022 & $7(23.3)$ & $3(6.4)$ & 0.041 \\
\hline Lymph node & $1(20.0)$ & $3(23.1)$ & $>0.999$ & $3(18.8)$ & $2(18.2)$ & $>0.999$ & $2(6.7)$ & $6(12.8)$ & 0.472 \\
\hline Other & 0 & $2(15.4)$ & $>0.999$ & $1(6.3)$ & $2(18.2)$ & 0.549 & $0(0)$ & $1(2.1)$ & 0.361 \\
\hline
\end{tabular}

Values are presented as number (\%). 


\section{DISCUSSION}

The prognostic difference between RSC and LSC has been reported since 2010 [13]. Large scale meta-analyses and multi-institutional studies have been performed for stage I-III colon cancer $[4-6,14]$. Petrelli et al. [4] reported that LSC was associated with a significantly reduced risk of death regardless of stage. However, Ishihara et al. [14] reported that the RFS of RSC was significantly better than LSC in stage II-III colon cancer. On the other hand, Ha et al. [5] and Kishiki et al. [6] reported that the prognosis of RSC was significantly worse in stage III colon cancer, while opposite results were obtained in stage I-II colon cancer. The present study analyzed difference between RSC and LSC in patients with stage II and stage III disease separately. As there were some significantly different clinicopathological factors, one-to-one propensity score matching was also performed. Regarding stage II disease, there was no significant prognostic difference between the RSC and LSC groups. On the other hand, OS and RFS of the RSC group were significantly worse in stage III disease in all cases and propensity-matched cases. Furthermore, multivariate analysis using Cox proportional hazard model revealed that the prognosis of the RSC group was significantly worse in stage III but not in stage II. Consistent with the previous reports, differences between stage II and III disease were observed in the present study. Although this study included a relatively small number of the patients, the treatment strategy and perioperative management were standardized. Moreover one-to-one propensity matching analysis provided more reliable results.

Based on a large-scale retrospective analysis, RSC was more frequently found in women and the elderly [13] and our study confirmed this, with more women in the RSC group with stage II disease, and significantly older patients in the RSC group with stage III disease. Previous reports have suggested that RSC were more advanced as they presented fewer symptoms with less opportunity for early diagnosis with colonoscopy compared with LSC $[6,13]$. However, in the present study, the proportion of the tumor stage T4 was significantly higher in the LSC group with stage II and III disease. This could have been because of referrals of advanced LSC to our institution as the high volume center in our region. RSC has been reported to include more poorly differentiated or mucinous histology $[5,13]$, and we found that was so in the RSC group with stage II disease.

Embryologically, the right colon derives from the midgut, while the left colon derives from the hindgut, and molecular and genetic differences between them have been documented. RSC more frequently have mutations of $R A S, B R A F$, and MSI-high [13, 15], while LSC more frequently have mutations of p53 and APC [16]. MSI-high was found to be more frequent in stage II RSC compared with stage III RSC [17], and was an independent favorable prognostic factor in colorectal cancer $[18,19]$. Mutations of $B R A F$ and $R A S$ have been reported to connect with aggressive tumor biology and poor prognosis. Therefore, the poor prognostic impact of mutations of $B R A F$ and $R A S$ in stage II RSC might be obscured by the impact of MSI-high.

In our study, the impact of primary tumor location on the prognosis after liver resection for CRLM was also studied. Clinicopathological factors were not significantly different between the RSC and LSC groups (except for the level of CA19-9), and propensitymatched analysis was not performed. The OS of the RSC group was significantly worse, while the RFS was not significantly different. Regarding metachronous liver metastasis, the patients whose primary colon cancer was stage II showed no significant difference of OS between RSC and LSC groups, while in the patients whose primary colon cancer was stage III, the OS of the RSC group was significantly worse than the LSC group. This result suggested the difference of tumor biology between stage II and stage III colon cancer which influenced even in metachronous liver metastasis. Although previous studies reported worse OS of RSC after liver resection for CRLM [9-12], the result focused on metachronous liver metastasis has not been reported yet. Moreover, the surgical indication and treatment strategy for CRLM were standardized in the present study [20-22]. Therefore, the results of the present study might be more reliable.

The details of sites of recurrence after liver resection were also analyzed in this study. Regarding liver metastasis, the RSC group included a significantly higher proportion of peritoneal dissemination $(23.3 \%$ vs. $6.4 \%, \mathrm{P}=0.041)$ which might have worsened OS of the RSC group, while not affecting RFS. Moreover, the proportion of peritoneal dissemination was significantly higher in the RSC group both in stage II and stage III. A correlation between $B R A F$ mutation and peritoneal recurrence has been reported [15]. Considering the high incidence of $B R A F$ mutation in RSC, these results were consistent with those findings.

The present study had limitations. First, it was retrospective in nature. Second, the study was from a single institution with a small sample size. Third, the assays of BRAF mutation, EGFR and MSI were not performed, and the number of assays for RAS mutation was very low (data not shown). Therefore, genetic assessment between the tumor location and the oncologic relevance was not performed in this study. Despite these limitations, the patients in this study underwent a standardized treatment strategy, with analysis by disease stage, and the propensity-matched analysis might strengthen the results.

In conclusion, sidedness of colon cancer had a significant and varied prognostic impact in patients with stage II, III, and liver metastasis after curative resection. RSCs had more peritoneal recurrence in each disease stage.

\section{CONFLICT OF INTEREST}

No potential conflict of interest relevant to this article was reported. 


\section{REFERENCES}

1. Ferlay J, Shin HR, Bray F, Forman D, Mathers C, Parkin DM. Estimates of worldwide burden of cancer in 2008: GLOBOCAN 2008. Int J Cancer 2010;127:2893-917.

2. Guinney J, Dienstmann R, Wang X, de Reyniès A, Schlicker A, Soneson C, et al. The consensus molecular subtypes of colorectal cancer. Nat Med 2015;21:1350-6.

3. Missiaglia E, Jacobs B, D’Ario G, Di Narzo AF, Soneson C, Budinska E, et al. Distal and proximal colon cancers differ in terms of molecular, pathological, and clinical features. Ann Oncol 2014;25: 1995-2001.

4. Petrelli F, Tomasello G, Borgonovo K, Ghidini M, Turati L, Dallera $\mathrm{P}$, et al. Prognostic survival associated with left-sided vs rightsided colon cancer: a systematic review and meta-analysis. JAMA Oncol 2017;3:211-9.

5. Ha GW, Kim JH, Lee MR. Oncologic effects of primary tumorsidedness on patients with stages 1-3 colon cancer: a meta-analysis. Ann Surg Oncol 2019;26:1366-75.

6. Kishiki T, Kuchta K, Matsuoka H, Kojima K, Asou N, Beniya A, et al. The impact of tumor location on the biological and oncological differences of colon cancer: multi-institutional propensity score-matched study. Am J Surg 2019;217:46-52.

7. Lim DR, Kuk JK, Kim T, Shin EJ. Comparison of oncological outcomes of right-sided colon cancer versus left-sided colon cancer after curative resection: which side is better outcome? Medicine (Baltimore) 2017;96:e8241.

8. Arnold D, Lueza B, Douillard JY, Peeters M, Lenz HJ, Venook A, et al. Prognostic and predictive value of primary tumour side in patients with RAS wild-type metastatic colorectal cancer treated with chemotherapy and EGFR directed antibodies in six randomized trials. Ann Oncol 2017;28:1713-29.

9. Yamashita S, Brudvik KW, Kopetz SE, Maru D, Clarke CN, Passot $\mathrm{G}$, et al. Embryonic origin of primary colon cancer predicts pathologic response and survival in patients undergoing resection for colon cancer liver metastases. Ann Surg 2018;267:514-20.

10. Russolillo N, Sperti E, Langella S, Menonna F, Allieta A, Di Maio $\mathrm{M}$, et al. Impact of primary tumor location on patterns of recurrence and survival of patients undergoing resection of liver metastases from colon cancer. HPB (Oxford) 2020;22:116-23.

11. Creasy JM, Sadot E, Koerkamp BG, Chou JF, Gonen M, Kemeny $\mathrm{NE}$, et al. The impact of primary tumor location on long-term survival in patients undergoing hepatic resection for metastatic colon cancer. Ann Surg Oncol 2018;25:431-8.

12. Dupré A, Malik HZ, Jones RP, Diaz-Nieto R, Fenwick SW, Poston GJ. Influence of the primary tumour location in patients undergoing surgery for colorectal liver metastases. Eur J Surg Oncol 2018;44:80-6.

13. Benedix F, Kube R, Meyer F, Schmidt U, Gastinger I, Lippert H, et al. Comparison of 17,641 patients with right- and left-sided colon cancer: differences in epidemiology, perioperative course, histology, and survival. Dis Colon Rectum 2010;53:57-64.

14. Ishihara S, Murono K, Sasaki K, Yasuda K, Otani K, Nishikawa T, et al. Impact of primary tumor location on postoperative recurrence and subsequent prognosis in nonmetastatic colon cancers: a multicenter retrospective study using a propensity score analysis. Ann Surg 2018;267:917-21.

15. Tran B, Kopetz S, Tie J, Gibbs P, Jiang ZQ, Lieu CH, et al. Impact of BRAF mutation and microsatellite instability on the pattern of metastatic spread and prognosis in metastatic colorectal cancer. Cancer 2011;117:4623-32.

16. Breivik J, Lothe RA, Meling GI, Rognum TO, Børresen-Dale AL, Gaudernack G. Different genetic pathways to proximal and distal colorectal cancer influenced by sex-related factors. Int J Cancer 1997;74:664-9.

17. Jernvall P, Mäkinen MJ, Karttunen TJ, Mäkelä J, Vihko P. Microsatellite instability: impact on cancer progression in proximal and distal colorectal cancers. Eur J Cancer 1999;35:197-201.

18. Malesci A, Laghi L, Bianchi P, Delconte G, Randolph A, Torri V, et al. Reduced likelihood of metastases in patients with microsatellite-unstable colorectal cancer. Clin Cancer Res 2007;13:3831-9.

19. Popat S, Hubner R, Houlston RS. Systematic review of microsatellite instability and colorectal cancer prognosis. J Clin Oncol 2005; 23:609-18.

20. Nakamoto T, Hokuto D, Nomi T, Yoshikawa T, Kamitani N, Matsuo $\mathrm{Y}$, et al. characteristics of five-year survivors after liver resection for colorectal liver metastases in modern chemotherapy. Anticancer Res 2020;40:1107-16.

21. Hokuto D, Nomi T, Yamato I, Yasuda S, Obara S, Yoshikawa T, et al. The prognosis of liver resection for patients with four or more colorectal liver metastases has not improved in the era of modern chemotherapy. J Surg Oncol 2016;114:959-65.

22. Hokuto D, Nomi T, Yasuda S, Yoshikawa T, Ishioka K, Yamada T, et al. Risk factors for unresectable recurrence after up-front surgery for colorectal liver metastasis. World J Surg 2018;42:884-91. 